\title{
Patch-seq: Past, Present, and Future
}

\author{
${ }^{\circledR}$ Marcela Lipovsek, ${ }^{1}{ }^{\circledR}$ Cedric Bardy, ${ }^{2,3}{ }^{\circledR}$ Cathryn R. Cadwell, ${ }^{4}{ }^{\circledR}$ Kristen Hadley, ${ }^{5}{ }^{\circledR}$ Dmitry Kobak, ${ }^{6}$ and \\ Shreejoy J. Tripathy ${ }^{7,8,9}$
}

${ }^{1}$ Centre for Developmental Neurobiology, Institute of Psychiatry, Psychology \& Neuroscience, King's College London, London SE1 1UL, United Kingdom, ${ }^{2}$ Laboratory for Human Neurophysiology and Genetics, South Australian Health and Medical Research Institute (SAHMRI), Adelaide 5000, SA, Australia, ${ }^{3}$ College of Medicine and Public Health, Flinders University, Bedford Park 5042, SA, Australia, ${ }^{4}$ Department of Pathology, University of California San Francisco, San Francisco, California 94143, ${ }^{5}$ Allen Institute for Brain Science, Seattle, Washington $98109,{ }^{6}$ Institute for Ophthalmic Research, University of Tübingen, 72076 Tübingen, Germany, ${ }^{7}$ Krembil Centre for Neuroinformatics, Centre for Addiction and Mental Health, Toronto, Ontario M5T 1R8, Canada, ${ }^{8}$ Department of Psychiatry, University of Toronto, Toronto, Ontario M5T 1R8, Canada, and ${ }^{9}$ Institute of Medical Science, University of Toronto, Toronto, Ontario M5S 1A8, Canada

Single-cell transcriptomic approaches are revolutionizing neuroscience. Integrating this wealth of data with morphology and physiology, for the comprehensive study of neuronal biology, requires multiplexing gene expression data with complementary techniques. To meet this need, multiple groups in parallel have developed "Patch-seq," a modification of whole-cell patchclamp protocols that enables mRNA sequencing of cell contents after electrophysiological recordings from individual neurons and morphologic reconstruction of the same cells. In this review, we first outline the critical technical developments that enabled robust Patch-seq experimental efforts and analytical solutions to interpret the rich multimodal data generated. We then review recent applications of Patch-seq that address novel and long-standing questions in neuroscience. These include the following: (1) targeted study of specific neuronal populations based on their anatomic location, functional properties, lineage, or a combination of these factors; (2) the compilation and integration of multimodal cell type atlases; and (3) the investigation of the molecular basis of morphologic and functional diversity. Finally, we highlight potential opportunities for further technical development and lines of research that may benefit from implementing the Patch-seq technique. As a multimodal approach at the intersection of molecular neurobiology and physiology, Patch-seq is uniquely positioned to directly link gene expression to brain function.

Key words: electrophysiology; transcriptomics; neuronal morphology; patch-clamp; single cell; multi-modal

\section{Introduction}

Single-cell approaches have been a cornerstone of neuroscience research for over a century, beginning with the Golgi staining (Golgi, 1873) and the exquisite descriptions of neuronal morphologies by Ramón y Cajal (1909; De Carlos and Borrell, 2007). Later on, the development of electrophysiological recording techniques allowed the study of individual neuronal responses

\footnotetext{
Received June 30, 2020; revised Nov. 11, 2020; accepted 0ct. 22, 2020.

The authors declare no competing financial interests.

M.L. was supported by a Consolidator Grant from the European Research Council (Grant 725729; FUNCOPLAN) to Matthew Grubb. C.B. was supported by the lan Potter Foundation, the Flinders Foundation, Impact Philanthropy, Cancer Australia, and the Australian Department of Health. K.H. was supported by the Allen Institute for Brain Sciences. D.K. was supported by the Deutsche Forschungsgemeinschaft (Grant BE5601/4-1 and the Cluster of Excellence "Machine Learning-New Perspectives for Science", EXC 2064, Project 390727645), the Federal Ministry of Education and Research (Grants FKZ 01G01601 and 01IS18039A), and the National Institute of Mental Health of the National Institutes of Health under Award Number U19MH114830. The content is solely the responsibility of the authors and does not necessarily represent the official views of the National Institutes of Health. S.J.T. was supported by the CAMH Discovery Fund, the Krembil Foundation, the Kavli Foundation, the Canadian Institutes of Health Research (Grant NGN-171423), and the Natural Sciences and Engineering Research Council of Canada (Grants RGPIN-2020-05834 and DGECR2020-00048). We thank Matthew Grubb for helpful comments on the manuscript and for kickstarting the organization of the mini-symposium.

Correspondence should be addressed to Marcela Lipovsek at marcela.lipovsek@kcl.ac.uk or Shreejoy J. Tripathy at shreejoy.tripathy@utoronto.ca.

https://doi.org/10.1523/JNEUROSCI.1653-20.2020

Copyright $\odot 2021$ the authors
}

(Hodgkin and Huxley, 1952; Hubel and Wiesel, 1959). Among these, the patch-clamp technique enabled isolated access to an individual neuron to study the ionic currents traversing the plasma membrane (Neher and Sakmann, 1976; Sakmann and Neher, 1984). Initial studies of the gene expression patterns of specific neuronal types relied on the purification of genetically labeled cells, followed by mRNA preparation and expression microarrays or RNA sequencing (Sugino et al., 2006; Okaty et al., 2009, 2011; Fishell and Rudy, 2011). These experiments looked at bulk samples of hundreds to millions cells, and so were limited by both the availability of known cell type-specific markers and the population average of gene expression levels. More recently, single-cell methods have entered the transcriptomic domain and revolutionized neuroscience, providing unparalleled insight into numerous neuronal features, such as cell type diversity (Zeisel et al., 2015, 2018; Gokce et al., 2016; Tasic et al., 2016, 2018; Stanley et al., 2020), developmental and differentiation trajectories (Habib et al., 2016; Kee et al., 2017; Nowakowski et al., 2017; Mi et al., 2018; Raj et al., 2018), and activity state (Chevée et al., 2018; Hrvatin et al., 2018; Jaeger et al., 2018; Sathyamurthy et al., 2018), all revealed by genome-wide gene expression patterns. However, the overall complexity and diversity of neurons often requires a combination of methodological approaches to address experimental questions. For example, recent developments in spatial transcriptomics have started profiling the expression of 
hundreds or thousands of genes in neurons, while preserving tissue architecture (Chen et al., 2015; Wang et al., 2018; Eng et al., 2019; Rodriques et al., 2019; Soldatov et al., 2019), and implementations of well established techniques for the combined evaluation of morphologic features and electrophysiological properties in the same individual neurons have been fundamental for improving our understanding of the cell types that are the building blocks of neuronal circuits (Jiang et al., 2015; Markram et al., 2015; Zeng and Sanes, 2017; Gouwens et al., 2019).

The study of genome-wide gene expression patterns concomitantly with other neuronal features has only recently become possible. Initial steps toward establishing direct links between electrophysiological properties and gene expression involved the profiling of a small number of genes using RT-PCR (Eberwine et al., 1992; Lambolez et al., 1992; Sucher and Deitcher, 1995; Chiang, 1998; Sucher et al., 2000; Toledo-Rodriguez et al., 2004; Pfeffer et al., 2013; Toledo-Rodriguez and Markram, 2014) or higher-throughput expression microarrays (Subkhankulova et al., 2010) on cytosolic aspirates sampled using the patch pipette. Though groundbreaking, these experiments were limited to small numbers of genes and/or cells. More recently, a number of groups have simultaneously developed Patch-seq, a multimodal method that combines electrophysiological recordings with single-cell whole-transcriptome profiling in the same individual cells (Fig. 1). The first attempt at single-cell RNA sequencing (scRNAseq) from neurons collected with a patch pipette profiled a very small number of samples (Table 1) and yielded sequencing data of low quality (Qiu et al., 2012). Improvements in scRNAseq (Picelli et al., 2014b) and adaptations of the patchclamp technique facilitated the successful and reliable implementation of Patch-seq (Bardy et al., 2016; Cadwell et al., 2016; Chen et al., 2016; Földy et al., 2016; Fuzik et al., 2016). Following these initial studies, the Patch-seq technique has been further optimized and applied to a number of model systems (Table 1). Moreover, these new types of experiments necessitated the development of innovative data analysis tools suitable for handling multimodal datasets (Kobak et al., 2018; Gala et al., 2019; Bernaerts et al., 2020).

In this review, we outline both experimental and analytical strategies for generating and interpreting high-quality Patch-seq data. We provide examples of new biological insights that have resulted from this transformative technique and further discuss outstanding questions especially well suited to the rich datasets produced by this multimodal approach.

\section{The Patch-seq experimental workflow}

Patch-seq experiments profile the electrophysiological properties and transcriptome of the same individual neurons, with the goal of identifying the underlying relationships between gene expression
Patch-seq on acute slices

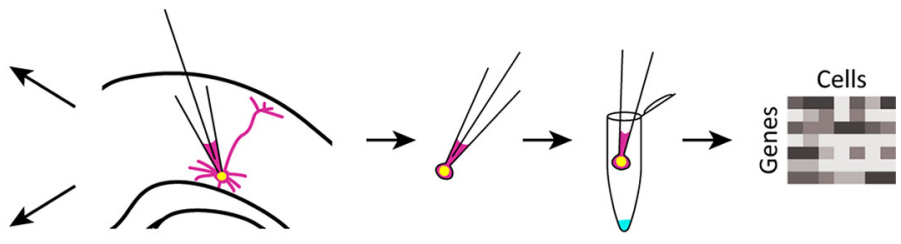

Patch-seq on cultured neurons

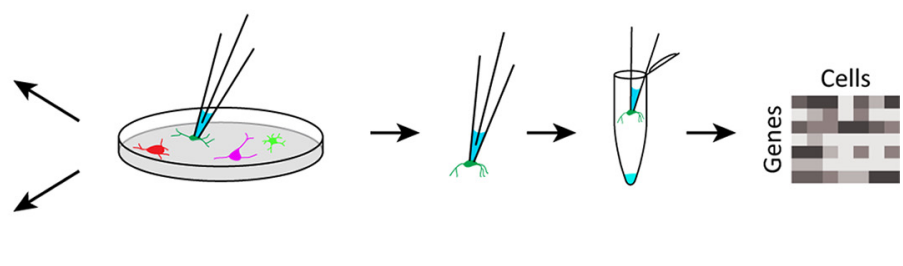

Patch-seq in vivo
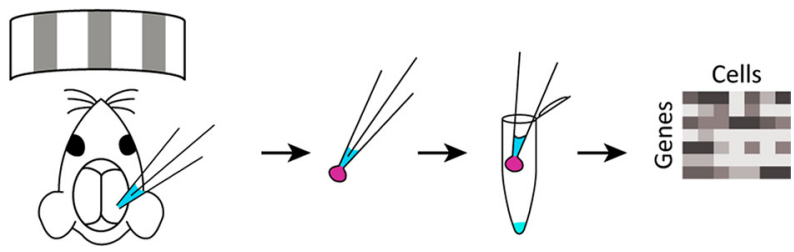

Figure 1. Schematic workflows of Patch-seq using various experimental model systems. $A, 0$ acute brain slices, electrophysiological recordings are performed on identified neurons, and the cell processes are filled with dye for subsequent morrecording and imaging, the whole cell is collected using the patch pipette and the transcriptome obtained via scRNAseq. $C$ In vivo patch-clamp recordings are used to study the responses of individual neurons to sensory stimuli. Using the patch pipette, each neuron can then be collected and processed for scRNAseq and for in vivo Patch-seq.

and neuronal function. Additionally, recorded neurons can be backfilled with appropriate dies to evaluate cell morphology.

\section{Sample collection}

Patch-seq experiments begin with whole-cell patch-clamp recordings from individual neurons to characterize their electrophysiological properties. There are a number of published protocols for Patch-seq (Table 1) that have been optimized for collecting mRNA from neurons recorded in acute brain slices (Cadwell et al., 2017; Lee et al., 2020; Lipovsek et al., 2020; Mahfooz and Ellender, 2021), or in vitro cell cultures (van den Hurk et al., 2018), and allowing subsequent morphologic reconstruction (Cadwell et al., 2017; van den Hurk et al., 2018; Scala et al., 2019, 2020; Gouwens et al., 2020; Que et al., 2020). Below, we discuss commonalities and differences between these protocols.

Given the ubiquitous nature of mRNA-degrading RNases in the environment and the small amount of mRNA present in each individual cell, Patch-seq protocols emphasize the importance of maintaining strict RNase-free conditions during sample collection and before reverse transcription (RT). This includes meticulously cleaning any surfaces that may come into contact with the mRNA sample or internal solution using 
Table 1. Summary of published Patch-seq datasets

\begin{tabular}{|c|c|c|c|c|c|c|}
\hline Publication & Dataset & Model system & Preparation & CDNA synthesis—library preparation & Total cells & Accession \\
\hline Qiu et al., 2012 & $\begin{array}{l}\text { Cultured hippocampal neurons - layer } 5 \\
\text { cortical neurons }\end{array}$ & Mouse & Primary cultures - acute slices & SMARTer-adapter ligation & 8 & NA \\
\hline Cadwell et al., 2016 & $\begin{array}{l}\text { Layer } 1 \text { cortical interneurons and pyramidal } \\
\text { neurons }\end{array}$ & Mouse & Acute slices and in vivo & Smart-seq2-Tagmentation & 58 & E-MTAB-4092 \\
\hline Fuzik et al., 2016 & $\begin{array}{l}\text { Layers } 1 \text { and } 2 \text { cortical interneurons and py- } \\
\text { ramidal neurons }\end{array}$ & Mouse & Acute slices & STRT-C1 (UMIs) —-Tagmentation & 80 & GSE70844 \\
\hline Chen et al., 2016 & Stem-cell derived neurons & Human & Cultured cells & (Tang et al., 2010) —NEBNext Ultra & 20 & GSE77564 \\
\hline Földy et al., 2016 & $\begin{array}{l}\text { Hippocampus pyramidal cells and } \\
\text { interneurons }\end{array}$ & Mouse & Acute slices & SMARTer-Tagmentation & 93 & GSE75386 \\
\hline Bardy et al., 2016 & iPSC-derived neurons and astrocytes & Human & Cultured cells & SMARTer-Tagmentation & 56 & GSE159074 \\
\hline Pfeffer and Beltramo, 2017 & $\begin{array}{l}\text { Interneurons and pyramidal neurons -Visual } \\
\text { cortex/ hippocampus }\end{array}$ & Mouse & Acute slices & CEL-Seq2 & 833 & GSE90822 \\
\hline Muñoz-Manchado et al., 2018 & Stratium interneurons & Mouse & Acute slices & STRT-C1 (UMIs) —-Tagmentation & 98 & GSE106708 \\
\hline Luo et al., 2019 & Hippocampus $\mathrm{VIP}^{+}$projecting interneurons & Mouse & Acute slices & SMART-Seq v4-Tagmentation & 8 & GSE109755 \\
\hline Wang et al., 2019 & $\begin{array}{l}\text { Temperature sensitive neurons - preoptic } \\
\text { area of the hypothalamus }\end{array}$ & Mouse & Acute slices & Smart-seq2-Tagmentation & 68 & GSE126657 \\
\hline Winterer et al., 2019 & Hippocampus interneurons & Mouse & Acute slices & SMART-Seq v4-Tagmentation & 46 & GSE124847 \\
\hline Oberst et al., 2019 & $\begin{array}{l}\text { Neuronal progenitors in developing mouse } \\
\text { neocortex }\end{array}$ & Mouse & Acute slices & SMART-Seq v4-Tagmentation & 65 & GSE122644 \\
\hline Scala et al., 2019 & Layer 4 sensory cortex neurons & Mouse & Acute slices & Smart-seq2-Tagmentation & 110 & GSE134378 \\
\hline Laboissonniere et al., 2019 & Retinal ganglion cells & Mouse & Acute dissection & SMART-Seq v4-Tagmentation & 14 & PRJNA548506 \\
\hline Ellender et al., 2019 & Somatosensory cortex excitatory neurons & Mouse & Acute slices & Smart-seq2-Tagmentation & 376 & NA \\
\hline Fuzik et al., 2019 & $\begin{array}{l}\text { Glutamatergic neurons of the indusium } \\
\text { griseum }\end{array}$ & Mouse & Acute slices & Smart-seq2-Tagmentation & NA & NA \\
\hline Kim et al., 2020b & Neurons from embryonic brain & Human & Acute slices & Modified aRNA—Illumina TrueSeq NuGEN Ovation & 1013 & GSE144216 \\
\hline Cadwell et al., 2020 & Clonally related cortical excitatory neurons & Mouse & Acute slices & Smart-seq2-Tagmentation & 220 & GSE140946 \\
\hline Liu et al., 2020 & Visual cortex light sensitive neurons & Mouse & In vivo & Smart-seq2_-adapter ligation & 53 & GSE115997 \\
\hline He et al., 2020 & Glucose (in)sensitive hypothalamic neurons & Mouse & Acute slices & SMART-Seq v4-Tagmentation & 8 & GSE146543 \\
\hline Camunas-Soler et al., 2020 & Pancreas islet cells & Human & Dispersed islet cells & Smart-seq2-Tagmentation & 1369 & GSE124742 \\
\hline Oláh et al., 2020 & Hippocampus $\mathrm{CCK}^{+}$interneurons & Rat & Acute slices & SMART-Seq v4-Tagmentation & 16 & GSE133951 \\
\hline Que et al., 2020 & Hippocampus $\mathrm{PV}^{+}$interneurons & Mouse & Acute slices & SMART-Seq v4-Tagmentation & 89 & GSE142546 \\
\hline Gouwens et al., 2020 & Visual cortex GABAergic neurons & Mouse & Acute slices & SMART-Seq v4-Tagmentation & 4270 & NEMO archive $^{\mathrm{a}}$ \\
\hline Scala et al., 2020 & $\begin{array}{l}\text { Excitatory and inhibitory neurons of the } \\
\text { motor cortex }\end{array}$ & Mouse & Acute slices & Smart-seq2-Tagmentation & 1221 & NEMO archive $^{b}$ \\
\hline Berg et al., 2020 & Cortical glutamatergic neurons & Human & Acute slices & SMART-Seq v4-Tagmentation & 385 & NA \\
\hline Bakken et al., 2020 & Primary motor cortex neurons & Marmoset Human & Acute slices & SMART-Seq v4-Tagmentation & 12 & NA \\
\hline Cadwell et al., 2017 & Cortical neurons & Mouse & Acute slices & Smart-seq2-Tagmentation & \multicolumn{2}{|c|}{ Protocol paper } \\
\hline van den Hurk et al., 2018 & iPSC-derived neurons & Human & Cultured cells & SMARTer-Tagmentation & \multicolumn{2}{|c|}{ Protocol paper } \\
\hline Lipovsek et al., 2020 & Dopaminergic neurons of the olfactory bulb & Mouse & Acute slices & Smart-seq2-Tagmentation & \multicolumn{2}{|c|}{ Protocol paper } \\
\hline Lee et al., 2020 & Primary visual cortex & Mouse & Acute slices & SMART-Seq v4-Tagmentation & \multicolumn{2}{|c|}{ Protocol paper } \\
\hline Mahfooz and Ellender, 2021 & Cortical neurons & Mouse & Acute slices & Smart-seq2-Tagmentation & \multicolumn{2}{|c|}{ Protocol book chapter } \\
\hline
\end{tabular}

an RNase decontamination solution and maintaining strict RNase-free conditions while preparing all pre-RT solutions.

Standard electrophysiological protocols can be performed in Patch-seq experiments to quantify the physiological properties of interest. The diameter of the recording pipette tip may need to be adjusted (typically, slightly larger tips are used; but see the study by Lipovsek et al., 2020) to facilitate the aspiration of cell contents into the pipette. Additionally, smaller than normal volumes of internal solution are used to avoid dilution of the RNA and reduce the surface area of glass in contact with the sample, which can lead to sample loss. Finally, the internal solution can be modified to further promote RNA preservation by adding calcium chelators, RNA carriers, and/or RNase inhibitors (Cadwell et al., 2017; van den Hurk et al., 2018; Lee et al., 2020; Lipovsek et al., 2020; Mahfooz and Ellender, 2021). In light of these modifications, the recordings may be more challenging compared with standard patch-clamp conditions and the overall recording time may need to be shortened to ensure minimal RNA degradation. In addition, the use of calcium chelators in the internal solution to reduce RNase activity (Sucher and Deitcher, 1995; Sucher et al., 2000) may also reduce the amplitude of calcium-dependent currents. With these caveats, there is no limit to the types of electrophysiological experiments that can be performed before aspiration of the cell contents for transcriptomic analysis.

After electrophysiological recordings are completed, the cytosol is aspirated into the recording pipette by applying negative pressure. The main objective is to collect the cell contents while maintaining a stable electrical seal between the pipette and the cell membrane, thus avoiding contamination from the extracellular solution. In recent protocols, it has been demonstrated that extracting both the cytosol and the nucleus, for example, using the nucleated patch technique, can greatly improve RNA yield and transcriptomic data quality (Gouwens et al., 2020; Lee et al., 2020; Lipovsek et al., 2020; Scala et al., 2020). After collection, the cell contents are deposited in individual tubes containing lysis and/or RT buffer. 
During recording, neurons can be filled with biocytin, enabling subsequent recovery of detailed cellular morphology (Cadwell et al., 2017; Fig. 1A). While this was not realized by the earliest applications of Patch-seq (Cadwell et al., 2016; Fuzik et al., 2016), recent adaptations to the internal and external solutions have substantially improved cell filling (Cadwell et al., 2017; Gouwens et al., 2020; Lee et al., 2020; Scala et al., 2020). Subsequent staining and morphologic reconstruction are further enhanced by the slow and careful retraction of the pipette during the sample collection step after electrophysiological recordings (Gouwens et al., 2020; Lee et al., 2020). Alternatively, if rhodamine is added to the intracellular solution, fluorescent images of the live neuron can be acquired before sample collection (Bardy et al., 2016; van den Hurk et al., 2018; Fig. 1B).

The potential applications of Patch-seq approaches span model systems, including traditional rodent models (Cadwell et al., 2016; Földy et al., 2016; Fuzik et al., 2016, 2019; MuñozManchado et al., 2018; Ellender et al., 2019; Laboissonniere et al., 2019; Oberst et al., 2019; Scala et al., 2019, 2020; Wang et al., 2019; Winterer et al., 2019; Cadwell et al., 2020; Gouwens et al., 2020; He et al., 2020; Liu et al., 2020; Oláh et al., 2020; Que et al., 2020), neurons derived from induced pluripotent stem cells (iPSCs; Bardy et al., 2016; Chen et al., 2016), and less genetically tractable models such as nonhuman primates (Bakken et al., 2020) and primary human tissue (Berg et al., 2020; Kim et al., 2020b). Patch-seq protocols have also been applied to non-neuronal pancreatic islet cells (Camunas-Soler et al., 2020). The basic procedure is very similar regardless of the model system, although the patching and sample collection techniques are tailored to the specific features of the tissue and cell type at hand (e.g., cell size, number of projections, age, myelinization). For example, the negative pressure applied to aspirate the cell contents needs to be carefully calibrated. In some instances (e. g., nonhuman primates and human tissue), cell viability is more variable, and so the extraction pressure needs to be adapted to each individual cell, with typically higher pressure applied for larger cell sizes. Also, if the neuron being extracted has a large number of projections embedded within the tissue, a careful balance between high and low negative pressure is needed to extract the cell contents without tearing the delicate processes.

The application of Patch-seq to the study of human iPSCderived neurons in vitro was developed independently (Bardy et al., 2016; Chen et al., 2016; van den Hurk et al., 2018) but shares features with Patch-seq methods tailored for acute brain slices (van den Hurk et al., 2018). The main advantages of the cell culture-based approach reside in the ability to perform high-resolution imaging of the live cells for morphology reconstructions and an enhanced capability to collect RNA from the entire neuron (Fig. $1 B$ ). The latter provides a more accurate representation of the complete transcriptional program of the cell, including mRNAs localized to synapses or dendrites (Cajigas et al., 2012), otherwise hidden in most other single-cell analyses that are restricted to the mRNA localized to the soma or the nucleus. Extraction of the complete cell (including soma and neurites) may prove more or less technically challenging depending on the stiffness of extracellular matrix, the size of the dendritic arborization, and neuronal adherence. In summary, Patch-seq is a versatile methodology, and the choice of protocol and model system depends on the experimental question at hand.

\section{cDNA synthesis, library preparation, and sequencing}

Having collected the contents from individually recorded neurons, subsequent steps aim to generate quantitative gene expression information using scRNAseq workflows. These involve cDNA synthesis and preparation of sequencing libraries. The protocol to be used is chosen before sample collection, as this will dictate the composition of the collection buffer. The cell contents aspirated using the patch recording pipette can be deposited into lysis buffer or on a buffer containing components for the RT reaction. Depending on the collection buffer and experimental workflow, RT reactions are performed on the day of recording or, following shortterm storage at $-80^{\circ} \mathrm{C}$, $\mathrm{RT}$ reactions can be performed in batches after sample collection is completed.

Patch-seq is not compatible with high-throughput dropletbased scRNAseq methods, such as $10 \times$ Genomics or Drop-seq (Macosko et al., 2015), but can be combined with tube/platebased methods for the preparation of cDNA and sequencing libraries. Synthetic mRNA spike-ins [e.g., ERCC (External RNA Controls Consortium) spike-ins; Jiang et al., 2011] can be included at this point, or in the internal solution used for electrophysiological recordings, to help control for technical variability. The most commonly used cDNA synthesis protocols are commercial (e.g., SMART-seq version 4, Clontech) or "off-the-shelf" versions of the popular Smart-Seq2 protocol (Picelli et al., 2014b). These are based on full-length cDNA synthesis and amplification using a template-switching oligonucleotide, followed by amplification of full-length cDNA. Other protocols allow for the incorporation of unique molecular identifiers (UMIs) followed by isolation of $5^{\prime}$-end fragments (Zeisel et al., 2015). The choice between full-length cDNA versus UMI-based approaches will depend on the experimental questions and data analysis workflow. The former is more sensitive and provides full-length coverage, allowing for analysis of splice variants (Ziegenhain et al., 2017), while the latter provides absolute numbers of molecule counts, correcting for library amplification bias. The newly developed Smart-Seq3 protocol combines full cDNA coverage and UMIs, greatly improving mRNA quantification and identification of isoforms (Hagemann-Jensen et al., 2020), and is compatible with Patch-seq workflows.

The preparation of sequencing libraries is usually performed using tagmentation-based methods, with either commercially available kits (e.g., Illumina Nextera XT) or off-the-shelf reagents (Picelli et al., 2014a,b). Both the cDNA and sequencing libraries undergo standard quality control steps. These may include capillary electrophoresis (e.g., Bioanalyzer or TapeStation) and quantification (e.g., Qubit) of amplified cDNA from individual cells (Cadwell et al., 2017; van den Hurk et al., 2018), as well as quantitative RT-PCR for housekeeping or marker genes and/or technical spike-ins (if included in the preparation; van den Hurk et al., 2018). Low-quality samples can be excluded from sequencing to conserve resources. Finally, short-read sequencing is typically performed on Illumina platforms. cDNA/library preparation and sequencing amount to most of the costs of running Patch-seq experiments. The choice between using commercially available kits or off-the-shelf reagents (see above) also greatly impacts overall expenses. For example, the cost of the SMART-seq version 4 kit (Takara) ranges from $\$ 40$ to $\$ 90$ per cell (excluding sequencing and equipment costs), whereas using off-the-shelf reagents can reduce the cost of library preparation to $\sim \$ 20$ per cell (Cadwell et al., 2017; Lee et al., 2020).

\section{Data processing and analysis}

Bioinformatics pipelines for low-level processing of Patch-seq data are typically identical to those currently in use for dissociated single-cell and single-nucleus RNA-seq data (Lun et al., 
2016; Luecken and Theis, 2019; Vieth et al., 2019). In brief, sequenced reads are first aligned to the reference genome and then quantified, typically at the gene level. In Patch-seq, up to $\sim 50 \%$ of reads come from unspliced transcripts (presumed to originate from the cell nucleus; Cadwell et al., 2017; Scala et al., 2020), similar to single-nucleus RNA-seq (Bakken et al., 2018), and so it can be beneficial to use both exonic and intronic read counts to quantify gene expression (Gouwens et al., 2020; Lee et al., 2020; Scala et al., 2020).

Following mapping and read quantification, there are a number of sample-level quality metrics that should be considered for Patch-seq. These include standard metrics commonly used for scRNAseq, such as the number of reads and genes detected per cell, the fraction of reads that map to the genome, the proportion of reads aligning to mitochondrial genes and spike-ins (if included), and the distribution of reads across intergenic, exonic, and intronic regions (Lun et al., 2016; Luecken and Theis, 2019; Vieth et al., 2019). These assessments are especially important when laboratories are in the early stages of implementing Patchseq, as the harvesting and sequencing of degraded mRNA often result in low-quality data. Additional quality control specific to Patch-seq involves quantifying mRNA contamination because of inadvertent sampling of mRNA from off-target sources, such as surrounding cells and neuropil (Tripathy et al., 2018; Gouwens et al., 2020; Lee et al., 2020; Scala et al., 2020). Using high-quality dissociation-based scRNAseq as a reference, noticeable glial contamination (e.g., microglial markers) has often been observed in Patch-seq samples obtained from acute brain slices. Computational approaches have been developed for quantifying and addressing the effect of these confounds in the context of downstream analyses (Tripathy et al., 2018; Lee et al., 2020).

Data analysis workflows will depend on the specific research questions being asked. When the objective is to study cell types, a common first step is to "map" Patch-seq transcriptomes to an existing transcriptomic reference atlas (Scala et al., 2019, 2020; Cadwell et al., 2020; Gouwens et al., 2020; Que et al., 2020). This is a key analysis step for classifying Patch-seq samples, as dissociation-based scRNAseq datasets typically encompass several orders of magnitude more cells and therefore provide more robust transcriptomic identification of cell types. Despite differences in expression profiles between Patch-seq and reference scRNAseq data, accurate mapping is typically possible using correlation-based methods (Fuzik et al., 2016; Stuart et al., 2019; Berg et al., 2020; Gouwens et al., 2020; Scala et al., 2020). This allows confident assignment of reference transcriptomic types to each Patch-seq cell and their positioning onto a reduced dimension (tSNE or UMAP) reference visualization (Kobak and Berens, 2019). Patch-seq cells with poor or inconsistent mapping to the reference atlas can then be discarded in an additional quality control step (Gouwens et al., 2020; Scala et al., 2020).

Finally, given the multimodal nature of Patch-seq data, there is strong interest in dedicated statistical tools for multimodal data exploration and visualization. Several methods relating gene expression to electrophysiological properties and allowing for low-dimensional visualizations have been developed, based on reduced-rank regression (Kobak et al., 2018), coupled autoencoders (Gala et al., 2019), and bottleneck networks (Bernaerts et al., 2020). It remains a challenge to include morphologic data in these statistical frameworks because of lower sample sizes and difficulties with adequately representing neural morphologies as feature vectors.

\section{Using Patch-seq to address novel biological questions}

\section{The advantages of targeted cell sampling}

Though relatively low throughput, the Patch-seq sample collection presents one crucial advantage over standard dissociationbased scRNAseq approaches: the experimenter knows exactly from which cell the transcriptomic sample is being collected (Fig. $2 A)$. This knowledge has been exploited to address multiple questions by pairing transcriptomes with spatial context, morphology, connectivity, and functional phenotypes (to name a few). For example, sample collection using the Patch-seq approach identified correlations between transcriptomes and neuronal position (Pfeffer and Beltramo, 2017; Gouwens et al., 2020; Scala et al., 2020), projection pattern, sensory response type (Pfeffer and Beltramo, 2017), and long-range axonal connectivity (Luo et al., 2019). The transcriptomes of individual cells identified solely by their functional response properties, such as thermosensitive neurons of the preoptic area of the hypothalamus (Wang et al., 2019), light-sensitive and insensitive neurons of the visual cortex (Liu et al., 2020), and glucose-inhibited/ excited neurons of the ventromedial hypothalamic nucleus ( $\mathrm{He}$ et al., 2020) have been contrasted, via Patch-seq, for the first time, revealing new molecular markers and genes involved in differential functional responses.

Targeted Patch-seq experiments also allow studying neurons with a shared developmental lineage. For example, Patch-seq revealed that cortical excitatory neurons derived from a common early progenitor are transcriptomically diverse and no more likely to belong to the same transcriptomic cell type than randomly selected neurons in similar anatomic locations (Cadwell et al., 2020). However, Patch-seq performed on excitatory neurons derived from different progenitor pools at later developmental stages revealed that apical intermediate progenitors contribute to a transcriptionally distinct layer $2 / 3$ neuronal subtype (Ellender et al., 2019). Finally, in combination with elegant transplantation experiments, sample collection via Patch-seq identified a prominent role for Wnt signaling in the temporal specification of apical neuronal progenitors (Oberst et al., 2019).

\section{A Rosetta stone for integrating multimodal atlases of cell type diversity}

The latest and largest scRNAseq studies of the mammalian brain describe hundreds of different transcriptomically defined cell types (La Manno et al., 2020; Yao et al., 2020), identified by clustering analysis. However, for many of these, the morphoelectric identity remains unknown. Patch-seq approaches are bridging this gap by directly profiling morphologic and electrophysiological phenotypes, alongside the transcriptomes, followed by mapping these multimodally defined cells onto comprehensive scRNAseq atlases (Fig. 2B). The first multimodal Patch-seq studies identified morphoelectrically and transcriptomically distinct neurons in layers 1 and 2 of the cortex (Cadwell et al., 2016; Fuzik et al., 2016). The latest multimodal classification efforts have profiled thousands of neurons in the mouse visual and motor cortices, comprising the majority of known transcriptomically defined cell types (Gouwens et al., 2020; Lee et al., 2020; Scala et al., 2020; Table 1).

The identification of distinct cell types is not always straightforward. In the striatum, transcriptomic classification of spiny projection neurons identified continuous as well as discrete variation between cell types (Gokce et al., 2016; Stanley et al., 2020). Application of Patch-seq to striatum interneurons uncovered a continuum of transcriptomic gradients that correlate with a 
continuum of electrophysiological phenotypes (Muñoz-Manchado et al., 2018), indicating that graded phenotypes may be a defining feature of neuronal diversity, at least in the striatum. In the hippocampus, Patch-seq of morphologically distinct parvalbumin-positive interneurons found them to be biophysically and transcriptionally homogeneous, but identified a developmental change in transcriptomic state that is characterized by the unexpected onset of hemoglobin expression (Que et al., 2020). Further exploiting the potential of Patchseq data, multimodal analysis refined the classification of layer 4 interneurons of the mouse visual and somatosensory cortex, suggesting that transcriptomically similar neurons can differ in terms of morphology and electrophysiology, depending on their cortical area (Scala et al., 2019).

The picture emerging from cross-modality studies is that no single feature is sufficient to unequivocally identify cell types. A study in the motor cortex suggested that, while broad families of transcriptomic cell types have distinct and essentially nonoverlapping morphoelectric phenotypes, closely related transcriptomic cell types are not well separated in the morphoelectric space (Scala et al., 2020). Also, corticocortical projection neurons of the visual cortex, identified as a single transcriptomically defined cell cluster, correspond to distinct cell types, defined by their anatomic projections and connectivity patterns (Kim et al., 2020a). Finally, continuous variation of morphologic, electrophysiological, and/or transcriptomic features between related cell types can limit the ability to predict one from the other, although using more than one modality can enhance prediction (Gouwens et al., 2020). The examples discussed above highlight the requirement for multimodal profiling to better determine cell identity and have crucial implications for the conceptual and empirical definitions of cell types, subtypes, and states (Zeng and Sanes, 2017).

\section{Uncovering the molecular basis of morphologic and functional diversity}

The unbiased nature of single-cell transcriptomics makes Patchseq ideal for generating novel hypotheses about the molecular underpinnings of morphologic and functional diversity. By targeting recordings to previously known functionally distinct cell types, the transcriptomic basis of such differences can now be potentially elucidated using Patch-seq (Wang et al., 2019; He et al., 2020; Liu et al., 2020). The predictive power of the Patch-seq approach to reveal features of neuronal function within local circuitry was illustrated in $\mathrm{CCK}^{+}$interneurons. Here, transcriptomic data identified differential expression of $5-\mathrm{HT}_{3}$ receptor subunits between interneuron types. At the functional level, this resulted in differential responses to serotonin and the further demonstration that, in interneurons that lack $5-\mathrm{HT}_{3}$ receptors mRNA, responses to serotonin are driven by gap junction coupling to neighboring cells (Fuzik et al., 2016). Similarly, Patchseq identified novel differentially expressed genes including cell adhesion and synaptic regulatory molecules, that may underlie differences in the connectivity of distinct subtypes of layer 1 interneurons (Cadwell et al., 2016).

Multimodal experiments have identified cell types with distinct functional or morphologic features that show highly similar transcriptomic profiles (Scala et al., 2019; Que et al., 2020; Kim et al., 2020a). In-depth analysis of one such example in the rat hippocampus found that $\mathrm{CCK}^{+}$interneurons of the CA3 area that display different excitability properties express distinct isoforms of voltage-gated potassium channel auxiliary subunits (Oláh et al., 2020). This demonstrates that subtle differences in transcriptomic profile can have profound consequences for neuronal function.

The potential for Patch-seq to predict functional states has also been demonstrated in human iPSC-derived neuronal cultures (Bardy et al., 2016). Such cultures often comprise neuronal populations of heterogeneous electrophysiological states or neurodevelopmental maturity. Applied to a Patch-seq dataset of iPSC-derived neurons, a machine-learning classifier efficiently predicted the electrophysiological state of individual neurons from their transcriptional profile (Fig. 2C). Once trained on Patch-seq data, such a classifier was applied to thousands of neurons profiled by high-throughput scRNAseq, allowing the study of functional heterogeneity in iPSC-derived cultures (Bardy et al., 2016). Similar machine learning approaches for predicting aspects of neuronal physiology from genome-wide gene expression have also been implemented on layer 1 cortical interneurons (Cadwell et al., 2016). Patch-seq can further be used to identify specific genes whose expression correlates strongly with electrophysiological properties (Fig. 2D). For example, mRNA level of Kcna1, which codes for subunits of the $\mathrm{K}_{\mathrm{v}} 1.1$ voltage-gated potassium channel, correlates strongly with cell-to-cell variability in action potential width among inhibitory cell types in the hippocampus and striatum (Bomkamp et al., 2019).

Finally, Patch-seq methods are not restricted to neurons. An extensive study of human pancreas samples combined electrophysiological measurements of exocytosis and channel activity 
with scRNAseq of pancreatic $\beta$-cells (Camunas-Soler et al., 2020). Subsequent analyses of cell function and gene expression networks identified a gene set associated with functional heterogeneity that can be used to predict endocrine physiology. The authors go further to demonstrate that Patch-seq can be used to study cryopreserved tissue (Camunas-Soler et al., 2020). The application of these novel approaches to patient samples holds great promise for future research and therapeutic avenues for the treatment of diabetes.

\section{Outstanding questions and future directions}

Patch-seq is a multimodal approach that can simultaneously profile multiple aspects of neuronal biology, namely their transcriptome, electrophysiological properties, anatomic location, and morphology. As such, it holds immense potential to address a multitude of outstanding questions in neuroscience. Approaches using Patch-seq have already made significant contributions to the identification of cell types and subtypes (Muñoz-Manchado et al., 2018; Gouwens et al., 2020; Scala et al., 2020), providing an essential backdrop for current and future neuroscience research.

The Patch-seq methodology presents three significant challenges. First, it is relatively low throughput, as the number of cells that can be profiled in a given experiment is governed by the practical considerations of patch-clamp electrophysiology (as opposed to the high throughput of droplet-based scRNAseq), made more cumbersome by the specific requirements of Patchseq sample collection. Second, Patch-seq is a laborious technique to learn and master. However, recent protocol improvements have substantially improved the success rates of critical mRNA sample collection and morphology recovery steps (Cadwell et al., 2017; Gouwens et al., 2020; Lee et al., 2020; Lipovsek et al., 2020; Scala et al., 2020). Last, Patch-seq requires interdisciplinarity, as it yields multiple types of data, each calling for their own skillset to analyze and interpret. Higher-throughput methods can individually profile the modalities targeted by Patch-seq. Using dedicated data analysis tools, these can be combined post hoc to start exploring their relationships (Tripathy et al., 2017; Butler et al., 2018). However, Patch-seq is unique in the sense that all data modalities are acquired from each individual cell simultaneously, providing unique and integrative opportunities for cross-modal data exploration.

Overall, Patch-seq is a versatile methodology and can be applied to any sample suitable for patch-clamp experiments. Decades of electrophysiological research provide virtually endless tried experimental model systems. Going beyond traditional acute mouse or rat brain slices, Patch-seq has already been implemented on neurosurgically resected human tissues to identify increased glutamatergic neuron diversity in the human cortex (Berg et al., 2020) and in a comparative study of mouse, marmoset, and human motor cortex (Bakken et al., 2018).

Improvement, refinement, and modifications of patch-clamp recordings and sequencing methodologies will undoubtedly extend the reach of Patch-seq methods. To date, Patch-seq has already been applied to in vivo recordings (Cadwell et al., 2016; Pfeffer and Beltramo, 2017; Liu et al., 2020), thus opening up the opportunity to co-opt invaluable experimental paradigms (e.g., learning and memory, sensory processing, and motor control) into multimodal workflows to ultimately study the relationships between gene expression and behavior. Furthermore, incorporating additional steps after sample collection to separate the mRNA from the rest of the cell contents (e.g., G\&T protocol for simultaneous genome and transcriptome profiling; Macaulay et al., 2016; Lipovsek et al., 2020) could provide the opportunity to study additional modalities. For example, chromatin accessibility or DNA methylation patterns can be profiled on the genomic DNA (Smallwood et al., 2014; Buenrostro et al., 2015) to investigate the epigenomic correlates of functional phenotypes, and advances in single-cell proteomics will enable assaying protein abundance levels (Labib and Kelley, 2020). Finally, Patch-seq could be combined with CRISPR-based gene expression perturbation experiments (Gomez et al., 2019) to simultaneously study the transcriptomic and functional effects of specific mutations at the single-cell level.

Future experimental questions will undoubtedly drive protocol innovations. Placed at the intersection between the functional and molecular domains, Patch-seq approaches are ideally suited to explore open questions on cell state dynamics. For a defined cell type, how is the functional state affected by external input? Which are the key genes that define a functional state? How does a change in functional state relate to changes in transcriptomic state? Addressing these questions will contribute to identifying the molecular players shaping the landscape of possible states a given cell can adopt during development, aging, and disease (Trapnell, 2015).

A yet untapped area of investigation for Patch-seq are the transcriptomic mechanisms of synapse function and plasticity. These experiments would require improvements in both recording conditions and scRNAseq methods to mitigate the effects on mRNA stability of extended recording periods and to capture transcriptomic changes related to mRNA transport and localization. Additionally, Patch-seq approaches can be a starting point for studying circuit assembly. For example, a description of a cell type-specific code of synaptic and cell adhesion molecules and its relationship with a functional phenotype could be used to predict circuit connectivity from single cell-level transcriptomic data (Földy et al., 2016). With the expansion of Patch-seq datasets, this approach can first be validated on known connected neurons and subsequently exploited to predict hitherto unknown connections. Finally, multi-Patch-seq experiments, in which pairs (or groups) of neurons are simultaneously recorded to determine their connectivity and subsequently collected for sequencing, would allow a direct correlation between gene expression and connectivity of transcriptomically and morphoelectrically defined neurons.

Another exciting area of investigation is using Patch-seq to disentangle the causality between molecules and function. For example, while many genes might appear correlated with a quantitative functional feature, such as an electrophysiological property (Cadwell et al., 2016; Földy et al., 2016), pinpointing which of these genes is likely to drive the causal relationship is a major goal of multimodal experimental approaches (Tripathy et al., 2017; Bomkamp et al., 2019). Addressing these questions will require further experimental manipulations alongside the development of new data analysis frameworks to distill understanding from complex multimodal datasets.

Overall, multimodal approaches to study cell types and function are becoming the norm, as new experimental and analytical tools are developed, allowing the exploration of long-standing and novel questions in neuroscience. Many of these approaches are focused on increasing the experimental throughput, aiming at profiling the highest number of cells possible. In parallel, lower-throughput approaches that are tailored to addressing specific questions can be equally or more powerful. Though Patchseq is a laborious approach, by directly interrogating the connections between organizational levels it is already proving invaluable for advancing our understanding of neuronal function. 


\section{References}

Bakken TE, Hodge RD, Miller JA, Yao Z, Nguyen TN, Aevermann B, Barkan E, Bertagnolli D, Casper T, Dee N, Garren E, Goldy J, Graybuck LT, Kroll M, Lasken RS, Lathia K, Parry S, Rimorin C, Scheuermann RH, Schork NJ, et al. (2018) Single-nucleus and single-cell transcriptomes compared in matched cortical cell types. PLoS One 13:e0209648.

Bakken TE, Jorstad NL, Hu Q, Lake BB, Tian W, Kalmbach BE, Crow M, Hodge RD, Krienen FM, Sorensen SA, Eggermont J, Yao Z, Aevermann $\mathrm{BD}$, Aldridge AI, Bartlett A, Bertagnolli D, Casper T, Castanon RG, Crichton K, Daigl TL, et al. (2020) Evolution of cellular diversity in primary motor cortex of human, marmoset monkey, and mouse. bioRxiv 016972. doi: https://doi.org/10.1101/2020.03.31.016972.

Bardy C, van den Hurk M, Kakaradov B, Erwin JA, Jaeger BN, Hernandez RV, Eames T, Paucar AA, Gorris M, Marchand C, Jappelli R, Barron J, Bryant AK, Kellogg M, Lasken RS, Rutten BPF, Steinbusch HWM, Yeo GW, Gage FH (2016) Predicting the functional states of human iPSCderived neurons with single-cell RNA-seq and electrophysiology. Mol Psychiatry 21:1573-1588.

Berg J, Sorensen SA, Ting JT, Miller JA, Chartrand T, Buchin A, Bakken TE, Budzillo A, Dee N, Ding S-L, Gouwens NW, Hodge RD, Kalmbach B, Lee C, Lee BR, Alfiler L, Baker K, Barkan E, Beller A, Berry K, et al. (2020) Human cortical expansion involves diversification and specialization of supragranular intratelencephalic-projecting neurons. bioRxiv 018820. doi: https://doi.org/10.1101/2020.03.31.018820.

Bernaerts Y, Berens P, Kobak D (2020) Sparse bottleneck networks for exploratory analysis and visualization of neural Patch-seq data. arXiv:2006.10411.

Bomkamp C, Tripathy SJ, Bengtsson Gonzales C, Hjerling-Leffler J, Craig AM, Pavlidis P (2019) Transcriptomic correlates of electrophysiological and morphological diversity within and across excitatory and inhibitory neuron classes. PLoS Comput Biol 15:e1007113.

Buenrostro JD, Wu B, Litzenburger UM, Ruff D, Gonzales ML, Snyder MP, Chang HY, Greenleaf WJ (2015) Single-cell chromatin accessibility reveals principles of regulatory variation. Nature 523:486-490.

Butler A, Hoffman P, Smibert P, Papalexi E, Satija R (2018) Integrating single-cell transcriptomic data across different conditions, technologies, and species. Nat Biotechnol 36:411-420.

Cadwell CR, Palasantza A, Jiang X, Berens P, Deng Q, Yilmaz M, Reimer J, Shen S, Bethge M, Tolias KF, Sandberg R, Tolias AS (2016) Electrophysiological, transcriptomic and morphologic profiling of single neurons using Patch-seq. Nat Biotechnol 34:199-203.

Cadwell CR, Scala F, Li S, Livrizzi G, Shen S, Sandberg R, Jiang X, Tolias AS (2017) Multimodal profiling of single-cell morphology, electrophysiology, and gene expression using Patch-seq. Nat Protoc 12:2531-2553.

Cadwell CR, Scala F, Fahey PG, Kobak D, Mulherkar S, Sinz FH, Papadopoulos S, Tan ZH, Johnsson P, Hartmanis L, Li S, Cotton RJ, Tolias KF, Sandberg R, Berens P, Jiang X, Tolias AS (2020) Cell type composition and circuit organization of clonally related excitatory neurons in the juvenile mouse neocortex. Elife 9:e52951.

Cajigas IJ, Tushev G, Will TJ, tom Dieck S, Fuerst N, Schuman EM (2012) The local transcriptome in the synaptic neuropil revealed by deep sequencing and high-resolution imaging. Neuron 74:453-466.

Camunas-Soler J, Dai X-Q, Hang Y, Bautista A, Lyon J, Suzuki K, Kim SK, Quake SR, MacDonald PE (2020) Patch-seq links single-cell transcriptomes to human islet dysfunction in diabetes. Cell Metab 31:1017-1031.e4.

Chen KH, Boettiger AN, Moffitt JR, Wang S, Zhuang X (2015) Spatially resolved, highly multiplexed RNA profiling in single cells. Science 348: aaa6090.

Chen X, Zhang K, Zhou L, Gao X, Wang J, Yao Y, He F, Luo Y, Yu Y, Li S, Cheng L, Sun YE (2016) Coupled electrophysiological recording and single cell transcriptome analyses revealed molecular mechanisms underlying neuronal maturation. Protein Cell 7:175-186.

Chevée M, Robertson JDJ, Cannon GH, Brown SP, Goff LA (2018) Variation in activity state, axonal projection, and position define the transcriptional identity of individual neocortical projection neurons. Cell Rep 22:441455.

Chiang LW (1998) Detection of gene expression in single neurons by patchclamp and single-cell reverse transcriptase polymerase chain reaction. J Chromatogr A 806:209-218.

De Carlos JA, Borrell J (2007) A historical reflection of the contributions of Cajal and Golgi to the foundations of neuroscience. Brain Res Rev $55: 8-16$.
Eberwine J, Yeh H, Miyashiro K, Cao Y, Nair S, Finnell R, Zettel M, Coleman P (1992) Analysis of gene expression in single live neurons. Proc Natl Acad Sci U S A 89:3010-3014.

Ellender TJ, Avery SV, Mahfooz K, Scaber J, von Klemperer A, Nixon SL, Buchan MJ, van Rheede JJ, Gatti A, Waites C, Pavlou HJ, Sims D, Newey SE, Akerman CJ (2019) Embryonic progenitor pools generate diversity in fine-scale excitatory cortical subnetworks. Nat Commun 10:5224.

Eng C-HL, Lawson M, Zhu Q, Dries R, Koulena N, Takei Y, Yun J, Cronin C, Karp C, Yuan G-C, Cai L (2019) Transcriptome-scale super-resolved imaging in tissues by RNA seqFISH. Nature 568:235-239.

Fishell G, Rudy B (2011) Mechanisms of inhibition within the telencephalon: "where the wild things are". Annu Rev Neurosci 34:535-567.

Földy C, Darmanis S, Aoto J, Malenka RC, Quake SR, Südhof TC (2016) Single-cell RNAseq reveals cell adhesion molecule profiles in electrophysiologically defined neurons. Proc Natl Acad Sci U S A 113:E5222E5231.

Fuzik J, Zeisel A, Máté Z, Calvigioni D, Yanagawa Y, Szabó G, Linnarsson S, Harkany T (2016) Integration of electrophysiological recordings with single-cell RNA-seq data identifies neuronal subtypes. Nat Biotechnol 34:175-183.

Fuzik J, Rehman S, Girach F, Miklosi AG, Korchynska S, Arque G, Romanov RA, Hanics J, Wagner L, Meletis K, Yanagawa Y, Kovacs GG, Alpár A, Hökfelt TGM, Harkany T (2019) Brain-wide genetic mapping identifies the indusium griseum as a prenatal target of pharmacologically unrelated psychostimulants. Proc Natl Acad Sci U S A 116:25958-25967.

Gala R, Gouwens N, Yao Z, Budzillo A, Penn O, Tasic B, Murphy G, Zeng H, Sümbül U (2019) A coupled autoencoder approach for multi-modal analysis of cell types. In: Advances in Neural Information Processing Systems 32 (Wallach $\mathrm{H}$, Larochelle $\mathrm{H}$, Beygelzimer A, Alché-Buc F, Fox E, Garnett R, eds), pp 9267-9276. Red Hook, NY: Curran Associates.

Gokce O, Stanley GM, Treutlein B, Neff NF, Camp JG, Malenka RC, Rothwell PE, Fuccillo MV, Südhof TC, Quake SR (2016) Cellular taxonomy of the mouse striatum as revealed by single-cell RNA-Seq. Cell Rep $16: 1126-1137$

Golgi C (1873) Sulla struttura della sostanza grigia del cervelo. Gazzetta Medica Italiana Lombardia 33:244-246.

Gomez JA, Beitnere U, Segal DJ (2019) Live-animal epigenome editing: convergence of novel techniques. Trends Genet 35:527-541.

Gouwens NW, Sorensen SA, Berg J, Lee C, Jarsky T, Ting J, Sunkin SM, Feng D, Anastassiou CA, Barkan E, Bickley K, Blesie N, Braun T, Brouner K, Budzillo A, Caldejon S, Casper T, Castelli D, Chong P, Crichton K, et al. (2019) Classification of electrophysiological and morphological neuron types in the mouse visual cortex. Nat Neurosci 22:1182-1195.

Gouwens NW, Sorensen SA, Baftizadeh F, Budzillo A, Lee BR, Jarsky T, Alfiler L, Baker K, Barkan E, Berry K, Bertagnolli D, Bickley K, Bomben J, Braun T, Brouner K, Casper T, Crichton K, Daigle TL, Dalley R, de Frates RA, et al. (2020) Integrated morphoelectric and transcriptomic classification of cortical GABAergic cells. Cell 183:935-953.e19.

Habib N, Li Y, Heidenreich M, Swiech L, Avraham-Davidi I, Trombetta JJ, Hession C, Zhang F, Regev A (2016) Div-Seq: single-nucleus RNA-Seq reveals dynamics of rare adult newborn neurons. Science 353:925-928.

Hagemann-Jensen M, Ziegenhain C, Chen P, Ramsköld D, Hendriks G-J, Larsson AJM, Faridani OR, Sandberg R (2020) Single-cell RNA counting at allele and isoform resolution using Smart-seq3. Nat Biotechnol 38:708-714.

He Y, Xu P, Wang C, Xia Y, Yu M, Yang Y, Yu K, Cai X, Qu N, Saito K, Wang J, Hyseni I, Robertson M, Piyarathna B, Gao M, Khan SA, Liu F, Chen R, Coarfa C, Zhao Z, et al. (2020) Estrogen receptor- $\alpha$ expressing neurons in the ventrolateral VMH regulate glucose balance. Nat Commun 11:2165.

Hodgkin AL, Huxley AF (1952) A quantitative description of membrane current and its application to conduction and excitation in nerve. J Physiol 117:500-544.

Hrvatin S, Hochbaum DR, Nagy MA, Cicconet M, Robertson K, Cheadle L, Zilionis R, Ratner A, Borges-Monroy R, Klein AM, Sabatini BL, Greenberg ME (2018) Single-cell analysis of experience-dependent transcriptomic states in the mouse visual cortex. Nat Neurosci 21:120-129.

Hubel DH, Wiesel TN (1959) Receptive fields of single neurones in the cat's striate cortex. J Physiol 148:574-591.

Jaeger BN, Linker SB, Parylak SL, Barron JJ, Gallina IS, Saavedra CD, Fitzpatrick C, Lim CK, Schafer ST, Lacar B, Jessberger S, Gage FH (2018) 
A novel environment-evoked transcriptional signature predicts reactivity in single dentate granule neurons. Nat Commun 9:3084.

Jiang L, Schlesinger F, Davis CA, Zhang Y, Li R, Salit M, Gingeras TR, Oliver B (2011) Synthetic spike-in standards for RNA-seq experiments. Genome Res 21:1543-1551.

Jiang X, Shen S, Cadwell CR, Berens P, Sinz F, Ecker AS, Patel S, Tolias AS (2015) Principles of connectivity among morphologically defined cell types in adult neocortex. Science 350:aac9462.

Kee N, Volakakis N, Kirkeby A, Dahl L, Storvall H, Nolbrant S, Lahti L, Björklund ÅK, Gillberg L, Joodmardi E, Sandberg R, Parmar M, Perlmann T (2017) Single-cell analysis reveals a close relationship between differentiating dopamine and subthalamic nucleus neuronal lineages. Cell Stem Cell 20:29-40.

Kim EJ, Zhang Z, Huang L, Ito-Cole T, Jacobs MW, Juavinett AL, Senturk G, Hu M, Ku M, Ecker JR, Callaway EM (2020a) Extraction of distinct neuronal cell types from within a genetically continuous population. Neuron 107:274-282.e6.

Kim JMH, Camarena A, Walker C, Lin MY, Wolseley V, Souaiaia T, Thornton M, Grubbs B, Chow RH, Evgrafov OV, Knowles JA (2020b) Robust RNA-Seq of aRNA-amplified single cell material collected by patch clamp. Sci Rep 10:1979.

Kobak D, Berens P (2019) The art of using t-SNE for single-cell transcriptomics. Nat Commun 10:5416.

Kobak D, Bernaerts Y, Weis MA, Scala F, Tolias A, Berens P (2018) Sparse reduced-rank regression for exploratory visualization of paired multivariate datasets. bioRxiv 302208. doi: https://doi.org/10.1101/302208.

Labib M, Kelley SO (2020) Single-cell analysis targeting the proteome. Nat Rev Chem 4:143-158.

Laboissonniere LA, Goetz JJ, Martin GM, Bi R, Lund TJS, Ellson L, Lynch MR, Mooney B, Wickham H, Liu P, Schwartz GW, Trimarchi JM (2019) Molecular signatures of retinal ganglion cells revealed through single cell profiling. Sci Rep 9:15778.

La Manno G, Siletti G, Furlan K, Gyllborg A, Vinsland D, Langseth CM E, Khven I, Johnsson A, Nilsson M, Lönnerberg P, Linnarsson S (2020) Molecular architecture of the developing mouse brain. bioRxiv 184051. doi: https://doi.org/10.1101/2020.07.02.184051.

Lambolez B, Audinat E, Bochet P, Crépel F, Rossier J (1992) AMPA receptor subunits expressed by single purkinje cells. Neuron 9:247-258

Lee BR, Budzillo A, Hadley K, Miller JA, Jarsky T, Baker K, Hill D, Kim L, Mann R, Ng L, Oldre A, Rajanbabu R, Trinh J, Braun T, Dalley R, Gouwens NW, Kalmbach BE, et al. (2020) Scaled high fidelity electrophysiological, morphological, and transcriptomic cell characterization. bioRxiv 369082. doi: https://doi.org/10.1101/2020.11.04.369082.

Lipovsek M, Browne L, Grubb MS (2020) Protocol for Patch-seq of small interneurons. STAR Protoc 100146.

Liu J, Wang M, Sun L, Pan NC, Zhang C, Zhang J, Zuo Z, He S, Wu Q, Wang X (2020) Integrative analysis of in vivo recording with single-cell RNA-seq data reveals molecular properties of light-sensitive neurons in mouse V1. Protein Cell 11:417-432.

Luecken MD, Theis FJ (2019) Current best practices in single-cell RNA-seq analysis: a tutorial. Mol Syst Biol 15:e8746.

Lun ATL, McCarthy DJ, Marioni JC (2016) A step-by-step workflow for lowlevel analysis of single-cell RNA-seq data with Bioconductor. F1000Res $5: 2122$.

Luo X, Muñoz-Pino E, Francavilla R, Vallée M, Droit A, Topolnik L (2019) Transcriptomic profile of the subiculum-projecting VIP GABAergic neurons in the mouse CA1 hippocampus. Brain Struct Funct 224:2269-2280.

Macaulay IC, Teng MJ, Haerty W, Kumar P, Ponting CP, Voet T (2016) Separation and parallel sequencing of the genomes and transcriptomes of single cells using G\&T-seq. Nat Protoc 11:2081-2103.

Macosko EZ, Basu A, Satija R, Nemesh J, Shekhar K, Goldman M, Tirosh I, Bialas AR, Kamitaki N, Martersteck EM, Trombetta JJ, Weitz DA, Sanes JR, Shalek AK, Regev A, McCarroll SA (2015) Highly parallel genomewide expression profiling of individual cells using nanoliter droplets. Cell 161:1202-1214.

Mahfooz K, Ellender TJ (2021) Combining whole-cell patch-clamp recordings with single-cell RNA sequencing. In: Patch clamp electrophysiology (Dallas M, Bell D, eds), pp 179-189. New York: Springer US.

Markram H, Muller E, Ramaswamy S, Reimann MW, Abdellah M, Sanchez CA, Ailamaki A, Alonso-Nanclares L, Antille N, Arsever S, Kahou GAA, Berger TK, Bilgili A, Buncic N, Chalimourda A, Chindemi G, Courcol J-
D, Delalondre F, Delattre V, Druckmann S, et al. (2015) Reconstruction and simulation of neocortical microcircuitry. Cell 163:456-492.

Mi D, Li Z, Lim L, Li M, Moissidis M, Yang Y, Gao T, Hu TX, Pratt T, Price DJ, Sestan N, Marín O (2018) Early emergence of cortical interneuron diversity in the mouse embryo. Science 360:81-85.

Muñoz-Manchado $A B$, Bengtsson Gonzales $C$, Zeisel A, Munguba $H$, Bekkouche B, Skene NG, Lönnerberg P, Ryge J, Harris KD, Linnarsson S, Hjerling-Leffler J (2018) Diversity of interneurons in the dorsal striatum revealed by single-cell RNA sequencing and PatchSeq. Cell Rep 24:21792190.e7.

Neher E, Sakmann B (1976) Single-channel currents recorded from membrane of denervated frog muscle fibres. Nature 260:799-802.

Nowakowski TJ, Bhaduri A, Pollen AA, Alvarado B, Mostajo-Radji MA, Di Lullo E, Haeussler M, Sandoval-Espinosa C, Liu SJ, Velmeshev D, Ounadjela JR, Shuga J, Wang X, Lim DA, West JA, Leyrat AA, Kent WJ, Kriegstein AR (2017) Spatiotemporal gene expression trajectories reveal developmental hierarchies of the human cortex. Science 358:1318-1323.

Oberst P, Fièvre S, Baumann N, Concetti C, Bartolini G, Jabaudon D (2019) Temporal plasticity of apical progenitors in the developing mouse neocortex. Nature 573:370-374

Okaty BW, Miller MN, Sugino K, Hempel CM, Nelson SB (2009) Transcriptional and electrophysiological maturation of neocortical fastspiking GABAergic interneurons. J Neurosci 29:7040-7052.

Okaty BW, Sugino K, Nelson SB (2011) Cell type-specific transcriptomics in the brain. J Neurosci 31:6939-6943.

Oláh VJ, Lukacsovich D, Winterer J, Arszovszki A, Lörincz A, Nusser Z, Földy C, Szabadics J (2020) Functional specification of CCK+ interneurons by alternative isoforms of Kv4.3 auxiliary subunits. Elife 9:e58515.

Pfeffer CK, Beltramo R (2017) Correlating anatomy and function with gene expression in individual neurons by combining in vivo labeling, patch clamp, and single cell RNA-seq. Front Cell Neurosci 11:376.

Pfeffer CK, Xue M, He M, Huang ZJ, Scanziani M (2013) Inhibition of inhibition in visual cortex: the logic of connections between molecularly distinct interneurons. Nat Neurosci 16:1068-1076.

Picelli S, Björklund ÅK, Reinius B, Sagasser S, Winberg G, Sandberg R (2014a) Tn5 transposase and tagmentation procedures for massively scaled sequencing projects. Genome Res 24:2033-2040.

Picelli S, Faridani OR, Björklund ÅK, Winberg G, Sagasser S, Sandberg R (2014b) Full-length RNA-seq from single cells using Smart-seq2. Nat Protoc 9:171-181.

Qiu S, Luo S, Evgrafov O, Li R, Schroth GP, Levitt P, Knowles JA, Wang K (2012) Single-neuron RNA-Seq: technical feasibility and reproducibility. Front Genet 3:124.

Que L, Lukacsovich D, Földy C (2020) Transcriptomic homogeneity and an age-dependent onset of hemoglobin expression characterize morphological PV types. bioRxiv 913103. doi: https://doi.org/10.1101/2020.01.21.913103.

Raj B, Wagner DE, McKenna A, Pandey S, Klein AM, Shendure J, Gagnon JA, Schier AF (2018) Simultaneous single-cell profiling of lineages and cell types in the vertebrate brain. Nat Biotechnol 36:442-450.

Ramón y Cajal S (1909) Histologie du système nerveux de l'homme \& des vertébrés. Paris: Maloine.

Rodriques SG, Stickels RR, Goeva A, Martin CA, Murray E, Vanderburg CR, Welch J, Chen LM, Chen F, Macosko EZ (2019) Slide-seq: a scalable technology for measuring genome-wide expression at high spatial resolution. Science 363:1463-1467.

Sakmann B, Neher E (1984) Patch clamp techniques for studying ionic channels in excitable membranes. Annu Rev Physiol 46:455-472.

Sathyamurthy A, Johnson KR, Matson KJE, Dobrott CI, Li L, Ryba AR, Bergman TB, Kelly MC, Kelley MW, Levine AJ (2018) Massively parallel single nucleus transcriptional profiling defines spinal cord neurons and their activity during behavior. Cell Rep 22:2216-2225.

Scala F, Kobak D, Shan S, Bernaerts Y, Laturnus S, Cadwell CR, Hartmanis L, Froudarakis E, Castro JR, Tan ZH, Papadopoulos S, Patel SS, Sandberg R, Berens P, Jiang X, Tolias AS (2019) Layer 4 of mouse neocortex differs in cell types and circuit organization between sensory areas. Nat Commun 10:4174.

Scala F, Kobak D, Bernabucci M, Bernaerts Y, Cadwell CR, Castro JR, Hartmanis L, Jiang X, Laturnus S, Miranda E, Mulherkar S, Tan ZH Yao Z, Zeng H, Sandberg R, Berens P, Tolias AS (2020) Phenotypic variation of transcriptomic cell types in mouse motor cortex. Nature. Advance online publication. Retrieved Nov 12, 2020. doi: 10.1038/s41586-0202907-3. 
Smallwood SA, Lee HJ, Angermueller C, Krueger F, Saadeh H, Peat J, Andrews SR, Stegle O, Reik W, Kelsey G (2014) Single-cell genome-wide bisulfite sequencing for assessing epigenetic heterogeneity. Nat Methods 11:817-820

Soldatov R, Kaucka M, Kastriti ME, Petersen J, Chontorotzea T, Englmaier L, Akkuratova N, Yang Y, Häring M, Dyachuk V, Bock C, Farlik M, Piacentino ML, Boismoreau F, Hilscher MM, Yokota C, Qian X, Nilsson M, Bronner ME, Croci L, et al. (2019) Spatiotemporal structure of cell fate decisions in murine neural crest. Science 364:eaas9536.

Stanley G, Gokce O, Malenka RC, Südhof TC, Quake SR (2020) Continuous and discrete neuron types of the adult murine striatum. Neuron 105:688699.e8.

Stuart T, Butler A, Hoffman P, Hafemeister C, Papalexi E, Mauck WM, Hao Y, Stoeckius M, Smibert P, Satija R (2019) Comprehensive integration of single-cell data. Cell 177:1888-1902.

Subkhankulova T, Yano K, Robinson HPC, Livesey FJ (2010) Grouping and classifying electrophysiologically-defined classes of neocortical neurons by single cell, whole-genome expression profiling. Front Mol Neurosci $3: 10$.

Sucher NJ, Deitcher DL (1995) PCR and patch-clamp analysis of single neurons. Neuron 14:1095-1100.

Sucher NJ, Deitcher DL, Baro DJ, Warrick RM, Guenther E (2000) Genes and channels: patch/voltage-clamp analysis and single-cell RT-PCR. Cell Tissue Res 302:295-307.

Sugino K, Hempel CM, Miller MN, Hattox AM, Shapiro P, Wu C, Huang ZJ, Nelson SB (2006) Molecular taxonomy of major neuronal classes in the adult mouse forebrain. Nat Neurosci 9:99-107.

Tang F, Barbacioru C, Nordman E, Li B, Xu N, Bashkirov VI, Lao K, Surani MA (2010) RNA-Seq analysis to capture the transcriptome landscape of a single cell. Nat Protoc 5:516-535.

Tasic B, Menon V, Nguyen TN, Kim TK, Jarsky T, Yao Z, Levi B, Gray LT, Sorensen SA, Dolbeare T, Bertagnolli D, Goldy J, Shapovalova N, Parry S, Lee C, Smith K, Bernard A, Madisen L, Sunkin SM, Hawrylycz M, et al. (2016) Adult mouse cortical cell taxonomy revealed by single cell transcriptomics. Nat Neurosci 19:335-346.

Tasic B, Yao Z, Graybuck LT, Smith KA, Nguyen TN, Bertagnolli D, Goldy J, Garren E, Economo MN, Viswanathan S, Penn O, Bakken T, Menon V, Miller J, Fong O, Hirokawa KE, Lathia K, Rimorin C, Tieu M, Larsen R, et al. (2018) Shared and distinct transcriptomic cell types across neocortical areas. Nature 563:72-78.

Toledo-Rodriguez M, Markram H (2014) Single-cell RT-PCR, a technique to decipher the electrical, anatomical, and genetic determinants of neuronal diversity. Methods Mol Biol 1183:143-158.

Toledo-Rodriguez M, Blumenfeld B, Wu C, Luo J, Attali B, Goodman P, Markram H (2004) Correlation maps allow neuronal electrical properties to be predicted from single-cell gene expression profiles in rat neocortex. Cereb Cortex 14:1310-1327.
Trapnell C (2015) Defining cell types and states with single-cell genomics. Genome Res 25:1491-1498.

Tripathy SJ, Toker L, Li B, Crichlow C-L, Tebaykin D, Mancarci BO, Pavlidis P (2017) Transcriptomic correlates of neuron electrophysiological diversity. PLoS Comput Biol 13:e1005814.

Tripathy SJ, Toker L, Bomkamp C, Mancarci BO, Belmadani M, Pavlidis P (2018) Assessing transcriptome quality in Patch-seq datasets. Front Mol Neurosci 11:363.

van den Hurk M, Erwin JA, Yeo GW, Gage FH, Bardy C (2018) Patch-seq protocol to analyze the electrophysiology, morphology and transcriptome of whole single neurons derived from human pluripotent stem cells. Front Mol Neurosci 11:261.

Vieth B, Parekh S, Ziegenhain C, Enard W, Hellmann I (2019) A systematic evaluation of single cell RNA-seq analysis pipelines. Nat Commun 10:4667.

Wang TA, Teo CF, Åkerblom M, Chen C, Tynan-La Fontaine M, Greiner VJ, Diaz A, McManus MT, Jan YN, Jan LY (2019) Thermoregulation via temperature-dependent PGD2 production in mouse preoptic area. Neuron 103:309-322.e7.

Wang X, Allen WE, Wright MA, Sylwestrak EL, Samusik N, Vesuna S, Evans K, Liu C, Ramakrishnan C, Liu J, Nolan GP, Bava F-A, Deisseroth K (2018) Three-dimensional intact-tissue sequencing of single-cell transcriptional states. Science 361:eaat5691.

Winterer J, Lukacsovich D, Que L, Sartori AM, Luo W, Földy C (2019) Single-cell RNA-Seq characterization of anatomically identified OLM interneurons in different transgenic mouse lines. Eur J Neurosci 50: 3750-3771.

Yao Z, Nguyen TC, van Velthoven CTJ, Goldy J, Sedeno-Cortes AE, Baftizadeh F, Bertagnolli D, Casper T, Crichton K, Ding SL, Fong O, Garren E, Glandon A, Gray J, Graybuck LT, Hirschstein D, Kroll M, Lathia K, Levi B, McMillen D, et al. (2020) A taxonomy of transcriptomic cell types across the isocortex and hippocampal formation. bioRxiv 015214. doi: https://doi.org/10.1101/2020.03.30.015214.

Zeisel A, Hochgerner H, Lönnerberg P, Johnsson A, Memic F, van der Zwan J, Häring M, Braun E, Borm LE, La Manno G, Codeluppi S, Furlan A, Lee K, Skene N, Harris KD, Hjerling-Leffler J, Arenas E, Ernfors P, Marklund U, Linnarsson S (2018) Molecular architecture of the mouse nervous system. Cell 174:999-1014.e22.

Zeisel A, Muñoz-Manchado AB, Codeluppi S, Lönnerberg P, La Manno G, Juréus A, Marques S, Munguba H, He L, Betsholtz C, Rolny C, CasteloBranco G, Hjerling-Leffler J, Linnarsson S (2015) Cell types in the mouse cortex and hippocampus revealed by single-cell RNA-seq. Science 347:1138-1142.

Zeng H, Sanes JR (2017) Neuronal cell-type classification: challenges, opportunities and the path forward. Nat Rev Neurosci 18:530-546.

Ziegenhain C, Vieth B, Parekh S, Reinius B, Guillaumet-Adkins A, Smets M, Leonhardt H, Heyn H, Hellmann I, Enard W (2017) Comparative analysis of single-cell RNA sequencing methods. Mol Cell 65:631-643.e4. 